\title{
FRACTIONAL BIORTHOGONAL PARTNERS IN FRACTIONALLY SPACED EQUALIZERS
}

\author{
Bojan Vrcelj and P. P. Vaidyanathan \\ Dept. of Electrical Engr. 136-93, Caltech, Pasadena, CA 91125, USA \\ E-mail: bojan@systems.caltech.edu,ppvnath@systems.caltech.edu
}

\begin{abstract}
The concept of fractional biorthogonal partners has been introduced recently by the authors. They arise in many different contexts, one of them being channel equalization with fractionally spaced equalizers. If the amount of oversampling at the receiver is not an integer, but a rational number, the problem of fractionally spaced equalization can be treated using the fractional biorthogonal partner setting. This approach is adopted here. We consider fractionally spaced equalizers with a rational amount of oversampling, show that the FIR solution (if it exists) is not unique and can be chosen to minimize the noise power at the receiver. These findings are demonstrated by examples where we compare the performance of fractionally spaced zero forcing equalizers to that of the corresponding minimum mean-squared error solution.
\end{abstract}

\section{INTRODUCTION}

Biorthogonal partners have been introduced by the authors in both the scalar [5] and the vector case [8]. A pair of digital filters $H(z)$ and $F(z)$ are called biorthogonal partners of each other with respect to an integer $M$ if their cascade $H(z) F(z)$ obeys the Nyquist $(M)$ property. This concept has been extended more recently [6] to the case where the upsampling and downsampling ratios are not integers but rational numbers. In that case, the filters are called fractional biorthogonal partners (FBPs) with respect to the ratio $L / M$. Construction of fractional biorthogonal partners is treated in [6] as well as conditions for the existence of FIR or just stable FBPs. One situation where FBPs arise has also been considered, namely signal interpolation.

In this paper we concentrate on another context in which FBPs have a natural application: that of channel equalization with fractionally spaced equalizers (FSEs). We show that, if the amount of oversampling at the receiver is a rational number, the problem can be posed in terms of fractional biorthogonal partners. The advantage is that we can employ the results developed in this and similar settings in order to find a fractionally spaced equalizer. Moreover, we will show that if an FIR solution exists, it is not unique and some optimization procedure can be used to construct a FSE that will reduce the noise power at the receiver.

We first give a brief overview of the communication systems with FSEs. Next, we review some of the results from [6] on fractional biorthogonal partners. We consider the nonuniqueness of FIR FBPs in greater detail, especially from the equalization point of view. After constructing the optimal FIR FBPs to be used as equalizers, we asses their performance in the section with experimental results.

Work supported in part by the ONR grant N00014-99-1-1002, USA.

\subsection{Notations}

If not stated otherwise, all notations are as in [4]. We use the encircled symbol $\downarrow M$ to denote the decimation operation [turns $x(n)$ into $x(M n)$ ]. The expanded version of $x(n)$

$$
\begin{cases}x(n / M) & \text { for } n=\text { mul of } M \\ 0 & \text { otherwise }\end{cases}
$$

is similarly obtained as a result of the expander operation which is denoted by the encircled symbol $\uparrow M$.

\section{FRACTIONALLY SPACED EQUALIZERS}

Consider the continuous time communication system shown in Fig. 1. Information sequence $x(n)$, with symbol spacing $T$ (rate $1 / T)$ is converted into an analog signal and after pulse shaping fed into the communication channel. This is shown in Fig. 1(a). Here $f_{c}(t)$ denotes the combined effect of the reconstruction filter from the D/A converter, pulse shaping filter as well as the continuous time channel. After passing through the channel, signal is corrupted with the additive noise and the received waveform $q_{c}(t)$ is sampled at the rate $(L / M) / T$ to produce the received sequence $q(n)$. If the ratio $L / M$ is equal to 1 , the equalizer at the receiver from Fig. 1(b) is called the symbol spaced equalizer (SSE). Several problems with this method have been pointed out in [3]. The receiver in this case becomes very sensitive to the phase shift at the sampling device; also, sampling at exactly the symbol rate may create some aliasing problems. That is why the preferred alternative is to keep $L>M$, giving rise to the receiver structure called the fractionally spaced equalizer (FSE) - see Fig. 1(b). The received sequence $q(n)$ with the denser spacing (higher rate) enters the fractionally spaced equalizer $h_{F S E}(n)$, which now has to operate at a slightly higher rate. Accompanied with this process, some rate reduction also needs to take place at the receiver, so that the final sequence $\hat{x}(n)$ entering the decision device has exactly the same rate $1 / T$ as the information sequence $x(n)$.

(a)

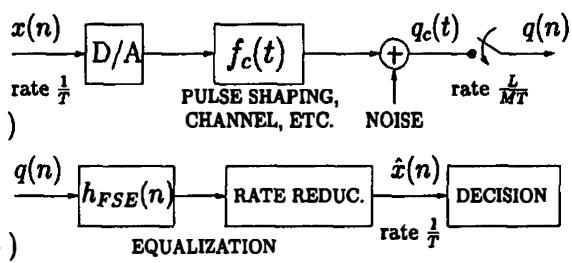

Fig. 1. Continuous time communication system. (a) Transmitter and channel. (b) Receiver. 


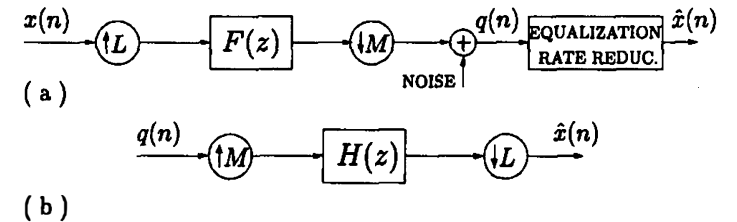

Fig. 2. FSEs with fractional oversampling. (a) Discrete time model of the communication system. (b) Form of the proposed equalizer.

The purpose of the FSE at the receiver is to compensate for the distortion introduced by $f_{c}(t)$. If the FSE is designed so that in the absence of noise $\hat{x}(n)=x(n)$, then it is called the zero-forcing equalizer (ZFE). Note, however that the ZFE is not necessarily the best solution, since we need to take into account the effect of the additive noise as well. In addition to taking care of some problems of SSEs mentioned earlier, FSEs often provide FIR zero-forcing solutions, which are in general favorable to IIR solutions for the reasons of stability and complexity of implementation. Moreover, in the case of vector signals and integer oversampling at the receiver (when $L / M$ is an integer) it has been shown [8] that the FIR solutions (even those of minimum order) are not unique. This flexibility in the design of vector ZFEs was utilized to further reduce the noise at the receiver $[7,8]$. Here we deal with the case where the oversampling ratio $L / M$ is not an integer but a rational number. This leads to FSEs with fractional oversampling, which are reviewed next. It is important to note here that if $L / M$ is just slightly greater than one, the computational overhead of the FSE with fractional oversampling is significantly smaller than that of the FSE with integer oversampling (since $L / M=2$ is the minimum oversampling ratio in the latter case).

\subsection{FSEs with fractional oversampling}

In the following we assume that $L>M$ and that $L$ and $M$ are coprime. Consider again Fig. 1(a) in the absence of noise. We can see that

$$
q(n)=q_{c}\left(n \frac{M}{L} T\right)=\sum_{k=-\infty}^{\infty} x(k) f_{c}\left(n \frac{M}{L} T-k T\right) .
$$

By defining the discrete time sequence $f(n) \triangleq f_{c}(n T / L)$, which is actually the function $f_{c}(t)$ sampled $L$ times more densely than at integers, we have

$$
q(n)=\sum_{k=-\infty}^{\infty} x(k) f(n M-k L) .
$$

This identity is incorporated in Fig. 2(a) where we show the discrete time model of the communication system from Fig. 1.

Obviously, the noise which is now discrete time needs to be modified with respect to the one in Fig. 1. The box labeled "equalization and rate reduction" is the object of our interest and we deal with it within the FBP setting.

\section{FRACTIONAL BIORTHOGONAL PARTNERS}

Let us first consider the problem of zero-forcing equalization. As mentioned before, this means that in the absence of noise the system drawn in Fig. 2(a) is identity. As motivated in [6], we look for the solution in the form shown in Fig. 2(b) and the complete system is presented in Fig. 3(a). For completeness we first provide the formal definition of fractional biorthogonal partners [6].

Definition. Transfer function $H(z)$ is said to be a right fractional biorthogonal partner (RFBP) of $F(z)$ with respect to the fraction $L / M$ if the system shown in Fig. 3(a) is identity in the absence of noise. Similarly, $F(z)$ is also said to be a left fractional biorthogonal partner (LFBP) of $H(z)$ with respect to $L / M$.

We conclude that our design problem is to find a stable, preferably FIR $H(z)$ given $F(z)$ and integers $L, M$ such that $H(z)$ is an RFBP of $F(z)$ with respect to $L / M$. Following the development from [6], we define the order- $L$ polyphase components of $F(z)$ and $H(z)$, namely $F_{k}(z)$ and $H_{k}(z)$ by

$$
F(z)=\sum_{k=0}^{L-1} F_{k}\left(z^{L}\right) z^{k}, \text { and } H(z)=\sum_{k=0}^{L-1} H_{k}\left(z^{L}\right) z^{-k} .
$$

Now, since $L$ and $M$ are coprime, we can find the integers $m$ and $l$ such that

$$
l L+m M=1 .
$$

In fact, the unique solution for the smallest $m$ and $l$ can be obtained by the Euclid's algorithm. Next we define the filters

$$
P_{k}(z) \triangleq z^{k l} F_{k}(z), \text { and } Q_{k}(z) \triangleq z^{-k l} H_{k}(z),
$$

for $0 \leq k \leq L-1$ and the order- $M$ polyphase components of these filters

$$
P_{k}(z)=\sum_{j=0}^{M-1} E_{k, j}\left(z^{M}\right) z^{-j}, \text { and } Q_{k}(z)=\sum_{i=0}^{M-1} R_{i, k}\left(z^{M}\right) z^{i} \text {. }
$$

It has been shown in [6] that the system from Fig. 3(a) is equivalent to the one in Fig. 3(b). Keeping in mind the definitions (3-6), the $L \times M$ analysis and the $M \times L$ synthesis polyphase matrices $\mathbf{E}(z)$ and $\mathbf{R}(z)$ respectively are given by

$$
\begin{aligned}
\mathbf{E}(z) & =\left[\begin{array}{cccc}
E_{0,0}(z) & E_{0,1}(z) & \cdots & E_{0, M-1}(z) \\
E_{1,0}(z) & E_{1,1}(z) & \cdots & E_{1, M-1}(z) \\
\vdots & \vdots & \vdots & \vdots \\
E_{L-1,0}(z) & E_{L-1,1}(z) & \cdots & E_{L-1, M-1}(z)
\end{array}\right], \\
\mathbf{R}(z) & =\left[\begin{array}{cccc}
R_{0,0}(z) & R_{0,1}(z) & \cdots & R_{0, L-1}(z) \\
R_{1,0}(z) & R_{1,1}(z) & \cdots & R_{1, L-1}(z) \\
\vdots & \vdots & \vdots & \vdots \\
R_{M-1,0}(z) & R_{M-1,1}(z) & \cdots & R_{M-1, L-1}(z)
\end{array}\right] .
\end{aligned}
$$

Note that given $F(z), L$ and $M$, matrix $\mathbf{E}(z)$ is uniquely defined. In order to find an RFBP of $F(z)$ we need to find a left inverse of $\mathbf{E}(z)$, namely $\mathbf{R}(z)$ in Fig. 3(b). Once we determined a suitable $\mathbf{R}(z)$, the corresponding RFBP $H(z)$ is again uniquely determined. This makes these two problems [shown in Fig. 3(a) and Fig. 3(b)] completely equivalent.

Under the assumption that $F(z)$ is FIR, we can easily see that $\mathbf{E}(z)$ is FIR as well. Of special interest is the situation when the RFBP $H(z)$ is FIR as well; in other words when the inverse $\mathbf{R}(z)$ is FIR. Notice that $\mathbf{E}(z)$ is a tall $L \times M$ polynomial matrix, so its left inverse can be FIR if and only if the gcd (greatest common divisor) [1] of all its $M \times M$ minors is a delay (for more details the reader is referred to [6]). 


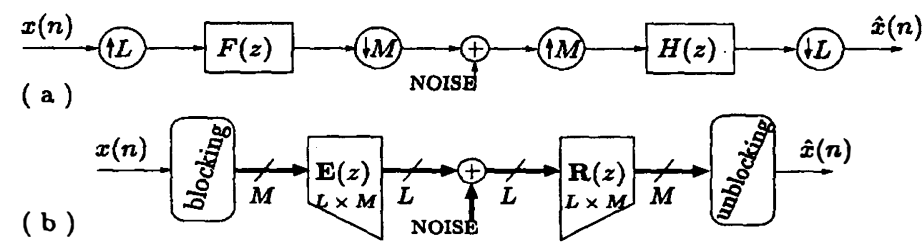

Fig. 3. Construction of FBPs. (a) Definition. (b) Equivalent form.

\subsection{Nonuniqueness of FIR RFBPs}

In the following we show that if the conditions for the existence of FIR solutions for $\mathbf{R}(z)$ are satisfied, this solution will not be unique. Furthermore, we use this nonuniqueness to construct a solution that performs favorably with respect to the undesired noise amplification at the receiver. More detailed treatment of a similar problem can be found in [7], so here we limit ourselves to just describing the solution. [1]

Let $\mathbf{E}(z)$ have an FIR left inverse and consider its Smith form

$$
\mathbf{E}(z)=\mathbf{U}(z) \boldsymbol{\Gamma}(z) \mathbf{V}(z)
$$

Here $\mathrm{U}(z)$ and $\mathrm{V}(z)$ are $L \times L$ and $M \times M$ unimodular matrices [1] and $\Gamma(z)$ is a $L \times M$ diagonal matrix. The elements on its diagonal are nonzero constants or delays, but without loss of generality we can assume that they are all constants. In other words, $\Gamma(z)=\left[\begin{array}{ll}\Gamma & 0\end{array}\right]^{T}$, where $\Gamma$ is a $M \times M$ constant diagonal matrix. Now from (8) we have that the general form ${ }^{1}$ of an FIR left inverse of $\mathbf{E}(z)$ is given by

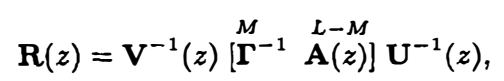

where $\mathbf{A}(z)$ is any $M \times(L-M)$ polynomial matrix. Note that any choice of $\mathbf{A}(z)$ will produce a valid FIR ZFE $H(z)$, but there will be an $\mathbf{A}(z)$ (of a given order $N_{A}$ ) that minimizes the noise component of $\hat{x}(n)$. In order to find such $\mathbf{A}(z)$ we consider the equivalent of Fig. 3(b) for the noise, shown in Fig. 4(a). Defining the polynomial matrices $D_{0}(z)$ and $D_{1}(z)$ to be

$$
[\underbrace{\mathrm{D}_{0}^{T}(z)}_{M} \underbrace{\mathrm{D}_{1}^{T}(z)}_{L-M}]^{T}=\mathrm{D}(z) \triangleq \mathrm{U}^{-1}(z)
$$

we can see that $\mathbf{R}(z)$ from (9) can be rewritten as

$$
\mathbf{R}(z)=\mathbf{V}^{-1}(z) \Gamma^{-1} \mathbf{D}_{0}(z)+\mathbf{V}^{-1}(z) \mathbf{A}(z) \mathbf{D}_{1}(z) .
$$

Defining $\mathbf{B}(z) \triangleq \mathbf{V}^{-1}(z) \mathbf{A}(z)$ we can now redraw Fig. 4(a) as in Fig. 4(b). The problem of finding the optimal $\mathbf{A}(z)$ is now transformed into the one of finding the optimal $\mathbf{B}(z)$ of order $N_{B}=N_{A}+\operatorname{ord}\left\{\mathbf{V}^{-1}(z)\right\}-1$. The solution can be found in the form of a Wiener filter [7]. Let $\mathbf{C}(z) \triangleq \mathbf{V}^{-1}(z) \Gamma^{-1} \mathrm{D}_{0}(z)$ and let the matrices $\mathbf{B}_{i}, \mathbf{C}_{i}$ and $\mathbf{D}_{i}$ represent the impulse responses of $\mathbf{B}(z), \mathbf{C}(z)$ and $\mathrm{D}_{1}(z)$ respectively. Next define the $M \times N_{C} L$ matrix $\mathcal{C}$ and the $(L-M) N_{B} \times L\left(N_{B}+N_{D}-1\right)$ matrix $\mathcal{D}_{1}$ as

$$
\mathcal{C} \triangleq\left[\begin{array}{llll}
\mathbf{C}_{0} & \mathbf{C}_{1} & \cdots & \mathbf{C}_{N_{C-1}}
\end{array}\right]
$$

\footnotetext{
${ }^{1}$ The unimodular matrices $U(z)$ and $V(z)$ in (8) are not unique so the form (9) can be made slightly more general.
}

(a)

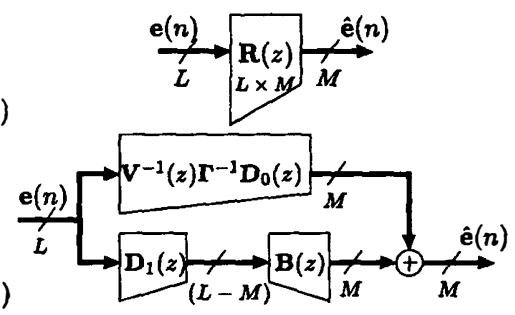

Fig. 4. (a)-(b) Finding the optimal FIR RFBP. See text.

$$
\mathcal{D}_{1} \triangleq\left[\begin{array}{cccccc}
\mathbf{D}_{0} & \ldots & \mathbf{D}_{N_{D}-1} & \mathbf{0} & \ldots & \mathbf{0} \\
\mathbf{0} & \mathbf{D}_{0} & \ldots & \mathbf{D}_{N_{D}-1} & \ldots & 0 \\
\vdots & & \ddots & & \ddots & \\
\mathbf{0} & \ldots & \mathbf{0} & \mathbf{D}_{0} & \ldots & \mathbf{D}_{N_{D^{-1}}}
\end{array}\right]
$$

Then the optimal $\mathbf{B}(z)$ is given by its impulse response matrix

$$
\begin{aligned}
\mathcal{B} & \triangleq\left[\begin{array}{llll}
\mathbf{B}_{0} & \mathbf{B}_{1} & \cdots & \mathbf{B}_{N_{B}-1}
\end{array}\right] \\
& =-\mathcal{C R}_{\epsilon}\left(1: N_{C} L,:\right) \mathcal{D}_{1}^{\dagger}\left(\mathcal{D}_{1} \mathcal{R}_{\epsilon}\left(1: N_{D}, 1: N_{D}\right) \mathcal{D}_{1}^{\dagger}\right)^{-1}(13)
\end{aligned}
$$

Here $\mathcal{R}_{\varepsilon}$ is a $L\left(N_{B}+N_{D}-1\right) \times L\left(N_{B}+N_{D}-1\right)$ autocorrelation matrix of the input noise process, and we use Matlab's notation $\mathbf{W}(1: N,:)$ to denote the matrix made of the first $N$ rows of $\mathbf{W}$.

\subsection{MMSE equalizer}

As we mentioned earlier, although the zero-forcing equalizer completely eliminates the channel distortion, the best equalizer $\mathbf{R}(z)$ of a given order $N_{R}-1$ in Fig. 3(b) is the one that minimizes the mean-squared error between $x(n)$ and $\hat{x}(n)$. This is nothing but the Wiener filter for vector signals described in [2]. Let the matrices $\mathbf{E}_{i}$ and $\mathbf{R}_{i}$ denote the impulse response of $\mathbf{E}(z)$ and $\mathbf{R}(z)$ respectively. Defining the $N_{R} L \times M\left(N_{R}+N_{E}-1\right)$ matrix $\mathcal{Q}$

$$
\mathcal{Q} \triangleq\left[\begin{array}{cccccc}
\mathbf{E}_{0} & \ldots & \mathbf{E}_{N_{E-1}} & \mathbf{0} & \ldots & \mathbf{0} \\
\mathbf{0} & \mathbf{E}_{0} & \ldots & \mathbf{E}_{N_{E-1}} & \ldots & \mathbf{0} \\
\vdots & & \ddots & & \ddots & \\
\mathbf{0} & \ldots & \mathbf{0} & \mathbf{E}_{0} & \ldots & \mathbf{E}_{N_{E}-1}
\end{array}\right]
$$

the MMSE solution for $\mathbf{R}(z)$ is given by its impulse response

$$
\begin{aligned}
\mathcal{P} & \triangleq\left[\begin{array}{llll}
\mathbf{R}_{0} & \mathbf{R}_{1} & \cdots & \mathbf{R}_{N_{R}-1}
\end{array}\right] \\
& =\mathcal{R}_{\mathcal{X X}}(1: M,:) \mathcal{Q}^{\dagger}\left(\mathcal{Q R}_{\mathcal{X X}} \mathcal{Q}^{\dagger}+\mathcal{R}_{e e}\right)^{-1}
\end{aligned}
$$



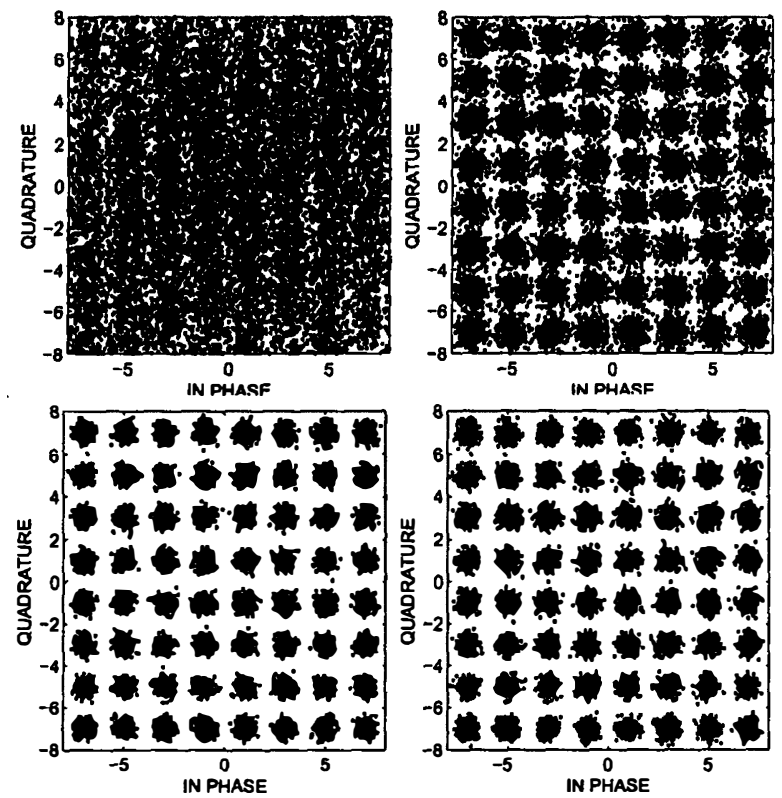

Fig. 5. Equalization results. Clockwise, starting from upper left: SSE, plain FIR RFBP, optimized FIR RFBP and MMSE methods.

Here $\mathcal{R}_{\mathcal{X} X}$ is a $M\left(N_{R}+N_{E}-1\right) \times M\left(N_{R}+N_{E}-1\right)$ autocorrelation matrix of the input sequence $x(n)$ and $\mathcal{R}_{e e}$ is a $N_{R} L \times N_{R} L$ autocorrelation matrix of the noise process.

Even though the MMSE method provides statistically the best solution, the equalizers based on zero-forcing are often preferred for simplicity reasons. Namely, comparing the two solutions (13) and (14) we see that as opposed to the MMSE method, the optimized FIR RFBP method does not require the knowledge of the signal autocorrelation matrix nor the noise variance.

\section{EXPERIMENTAL RESULTS}

In our experiments we compared the results of equalization of the iid input sequence $x(n)$ coming from a 64-QAM constellation using the four different methods; (1) traditional IIR SSE (case when $L=M$ ) and three FSE solutions: (2) the plain FIR RFBP method described in Sec. 3 [without the optimization matrix $\mathbf{A}(z)$ ], (3) optimized FIR RFBP method described in Sec. 3.1 and (4) the MMSE equalizer described in Sec. 3.2. The corresponding scattering diagrams are shown in Fig. 5. The channel sampled at integers was of the fourth order given by the coefficients

$$
\begin{array}{lllll}
1.0000 & 0.6600 & -0.3835 & -0.1276 & 0.5525
\end{array}
$$

and the corresponding sequence $f(n)\left[f_{c}(t)\right.$ oversampled by $\left.L\right]$ was obtained by linear interpolation. In the FSE implementations we took $L=5$ and $M=4$, so that the amount of computational overhead for the fractionally spaced equalizer (with respect to the symbol spaced one) was just $25 \%$. The order of the matrix $B(z)$ used in the optimized FIR RFBP method was $N_{B}-1=3$ and the order the Wiener solution $\mathbf{R}(z)$ given by (14) was $N_{R}-1=7$. For faimess, these were chosen so that both the optimized FIR RFBP and the MMSE equalizer have the same order. The noise was taken to be white and the SNR corresponding to Fig. 5 was 29

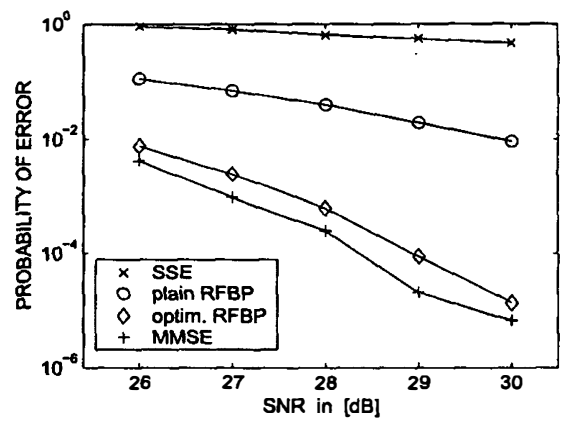

Fig. 6. Probability of error as a function of SNR in the four methods of equalization.

$\mathrm{dB}$. The obtained probabilities of error for the four methods (clockwise in Fig. 5) were $0.552,0.019,7.67 \times 10^{-5}$ and $2.00 \times 10^{-5}$ respectively. These examples show that the improvement in performance achieved by exploiting the redundancy in the construction of FIR RFBP is significant. It can also be observed that the method of optimized FIR RFBP equalizers does not perform far from the optimal MMSE equalizer of the same order, while it requires no knowledge of the input statistics and the noise variance.

\section{CONCLUDING REMARKS}

In this paper we consider one application fractional biorthogonal partners (FBPs), namely fractionally spaced equalization of the communication channels with a fractional oversampling at the receiver. The tools derived previously in the FBP setting prove useful in finding FIR zero-forcing fractionally spaced equalizers. We also show that this FIR equalization method allows for additional flexibility in construction, which can lead to significant improvements in the equalizer performance. Comparison with the MMSE equalizer shows no significant loss in performance, while the required knowledge of the system parameters is greatly reduced.

\section{REFERENCES}

[1] T. Kailath, Linear Systems. Prentice Hall, Inc., Englewood Cliffs, N.J., 1980.

[2] C. W. Therrien, Discrete Random Signals and Statistical Signal Processing. Prentice-Hall, Englewood Cliffs, NJ, 1992.

[3] J. R. Treichler, I. Fijalkow and C. R. Johnson, Jr., "Fractionally spaced equalizers: how long should they really be?," IEEE Signal Processing Magazine, pp. 65-81, May 1996.

[4] P. P. Vaidyanathan, Multirate Systems and Filter Banks. Prentice-Hall, Englewood Cliffs, NJ, 1995.

[5] P. P. Vaidyanathan and B. Vrcelj, "Biorthogonal partners and applications," IEEE Trans. Signal Processing, vol. 49(5), pp. 1013-1027, May 2001.

[6] B. Vrcelj and P. P. Vaidyanathan, "Fractional biorthogonal partners and application to signal interpolation," submitted to ISCAS 2002.

[7] B. Vrcelj and P. P. Vaidyanathan, "On the general form of FIR MIMO biorthogonal partners," Proc. 35th Asilomar Conf. on Sig., Sys. and Comp., Pacific Grove, CA, Nov. 2001.

[8] B. Vrcelj and P. P. Vaidyanathan, "Theory of MIMO biorthogonal partners and their application in channel equalization," Proceedings ICC, Helsinki, Finland, June 2001. 